\title{
Dietary factors and dental caries
}

\section{Can dietary measures assist in the prevention of dental caries?}

\section{Lingstrom P, Holm AK, Mejare I, et al. Dietary factors in the prevention of dental caries: a systematic review. Acta Odontol Scand 2003; 61:331-340}

Data sources Articles were sourced using Medline, the Cochrane Library, reference lists of identified articles and selected textbooks.

Study selection Studies chosen for inclusion in the review were randomised or controlled clinical trials of at least 2 years' duration that used caries increment in the permanent or primary dentition as the end point. Publications in Danish, English, French, German, Italian, Norwegian, Spanish or Swedish were included. For multiply reported trials the one with the longest follow-up period was included.

Data extraction and synthesis Inclusion decisions and grading of the studies was carried out independently by two of the authors. The main outcome was caries increment and the measure of treatment effect was either relative risk reduction or prevented faction. A qualitative synthesis of the included studies was conducted.

Results Eighteen studies met the inclusion criteria. They included the total or partial substitution of sucrose with sugar substitutes or the addition of protective foods to chewing gum. No study could be found that had evaluated the effect of information designed to reduce sugar intake/frequency as a single preventive measure. It is suggested that the evidence for the use of sorbitol or xylitol in chewing gum, or for the use of invert sugar, is inconclusive. No caries-preventive effect was found from adding calcium phosphate or dicalcium phosphate dihydrate to chewing gums.

Conclusions The review dearly demonstrates the need for welldesigned randomised clinical studies, with adequate control groups and high compliance, looking at the effect of dietary measures on dental caries.
Address for correspondence: Dr P Lingstrom, Department of Cariology, Faculty of Odontology, Sahlgrenska Academy, Göteborg University, Göteborg, Sweden. E-mail: lingstrom@odontologi.gu.se

\section{Commentary}

The importance of dietary factors in the aetiology of dental caries has long been acknowledged. Indeed, there is a professional consensus of opinion on the content of the preventive advice that should be provided to patients by the dental team. ${ }^{1}$ In their 1997 review of effectiveness, however, Kay and Locker ${ }^{2}$ stated that there was "no available evidence that oral health promotion is able to affect dietary practices to an extent whereby caries levels are reduced". By contrast, they pointed to the relative strength of evidence supporting the use of fluoride in caries reduction. Subsequent to this review, Blinkhorn ${ }^{3}$ was prompted to suggest that the dental profession had been overoptimistic about their ability to change dietary habits, and had failed to appreciate the importance of long-term interventions that offered regular reinforcement.

Looking at the broader context today, the role of good nutrition in improving the health of the population cannot be disputed. Setting out its new approach to public health in England, the Government has identified a number of overarching priorities for action. These include improving diet and nutrition which, although responding to the rapid increase in child and adult obesity, will also help to tackle heart disease, cancer, diabetes, stroke, high blood pressure and high cholesterol. ${ }^{4}$ Furthermore, the new clinical guideline for England and Wales on dental-recall intervals ${ }^{5}$ states that the dental team should cover diet (along with oral hygiene, fluoride, tobacco and alcohol use) in the initial comprehensive oral health assessment and subsequent oral health reviews, where relevant. The checklist of risk factors for each patient includes not only high and/or frequent sugar intake but also high and/or frequent dietary acid intake. More specifically, the long-term use of medications containing glucose, fructose or sucrose is highlighted, as is the erosive and cariogenic potential of the many soft drinks that are acidic and contain considerable amounts of simple sugars.

The guideline also recommends that research is carried out on the long-term clinical- and cost-effectiveness of one-to-one oral health advice and on a range of factors influencing this, such as the frequency with which it is delivered, the characteristics of the individual patient, and the medium and personnel employed to deliver the advice.

Does this review article add anything new to the existing literature, whether on the clinical evidence on the causation and prevention of dental caries, or the vexed issue of where the dental team should concentrate their preventive energies for greatest impact?

A systematic review can only be as informative as the quality and quantity of papers that can be included within its scope allow. Only 18 papers met the inclusion criteria for a randomised or controlled clinical trial, that is, at least 2 years' follow-up and caries increment as a primary end point. Disappointingly, no study was found that had evaluated the effect of information designed to reduce sugar intake/frequency as a single preventive measure. The gap in the evidence identified by Kay and Locker ${ }^{2}$ remains, therefore: the authors concluded that, "there is no evidence today to suggest that information to reduce sugar intake is an effective activity". 
Turning to the five studies included that evaluated sorbitol and xylitol, with a moderate level of evidence, the reviewers found the results were too limited for conclusions to be drawn. They therefore state that, "studies showing a caries-preventive effect from using sugar alcohols as a substitute for sucrose in chewing gums or candies/lozenges cannot be generalized...the hypothesis that xylitol has a superior role as a sugar substitute has not been verified". Further research is thus required. This contrasts with the conclusions of Macquire and Rugg-Gunn ${ }^{6}$ who state that chewing xylitol gum is certainly effective at preventing caries development compared with chewing sugared gum or no gum at all. Their paper also maintains that xylitol's specific effects on oral flora and especially on certain strains of mutans streptococci add to its caries-preventive profile and give it a unique role in preventive strategies for dental health. Edgar $^{7}$ has also reviewed the role of sugar-free gum, and suggests that its anticaries effects are worthwhile additional components of the home-based preventive regime, alongside fluoride use, oral hygiene and dietary control.

Where does this leave the confused dental practitioner, who has to offer chairside advice to patients, but who cannot wait for further (good-quality) research to be conducted and published, providing a more solid evidence-base?

The review does not set out to offer any practical advice on how to help and support individual patients in the dental surgery. Fortunately, Moynihan ${ }^{8}$ and Watt and McGlone ${ }^{9}$ have provided guidance for the dental team on dietary advice, which can be recommended. Those wishing to study the subject in greater depth can turn with confidence to Rugg-Gunn and Nunn. ${ }^{10}$ In addition, Daly et $a l^{11}$ have provided common-sense advice on how to approach behaviour change in general in the surgery, examining the process of change, motivations to change and barriers preventing change. Finally, Anderson's ${ }^{12}$ research has suggested that for dietary interventions within general dental practice, targeting children (especially relating to sweets, drinks and high sugar foods) would appear to be the best route forward. Anderson concludes that there is scope for dentists to be involved in initiating and reinforcing dietary messages and these may be expanded on by support staff or written resources. For the future, however, further work is required on defining cost-effective, practical, dietary interventions and funding routes.

To sum up, there are clear implications arising from this paper and the others discussed above:

- Although there is a lack of evidence on which to base their interventions, dental practitioners in England and Wales, at least, are unlikely to have the option of avoiding the provision of dietary advice to patients if they are to follow the relevant National Institute of Clinical Excellence guideline. ${ }^{5}$ In addition, the Chief Dental Officer for England's Delivering Change report ${ }^{13}$ anticipates dentists refocusing their care to disease prevention. In this scenario, primary care trusts will be able to work with dentists to provide patients with specific advice on diet and nutrition, taking into account cultural and religious beliefs.

- The dental team should ensure that they are up to date with current nutrition guidelines, both relating to general health, as set out by the English Food Standards Agency (see www.eatwell. gov.uk), for example, and relating to the causes and prevention of dental decay and dental erosion.

- Following published practical recommendations ${ }^{8,9,11}$ on dietary advice and behaviour change can assist the application of this scientific knowledge in the surgery setting.
- Ideally, the above information would be assembled in an easy-todigest form for the dental team to use for training purposes, in the same way that the Health Development Agency has provided a guide on smoking cessation specifically tailored to the needs and roles within the dental team. ${ }^{14}$ The lessons to be learnt from the scientific literature relating to interventions in the field of nutrition more broadly could also be usefully incorporated. A model is already available in the booklet provided by the Eastern Health and Social Services Board in Northern Ireland ${ }^{15}$ and in the integrated approach to the subject taken by Watt and McGlone. ${ }^{8}$

- The UK Government should consider carefully the recommendation made by the National Institute for Clinical Excellence ${ }^{5}$ and echoed by Anderson, ${ }^{12}$ that further research be carried out on the most effective delivery of one-to-one dietary advice. Seven years ago, Blinkhorn ${ }^{3}$ noted that, "for one-to-one education in the dental surgery it is essential to investigate just what our patients will cope with and understand when offered advice". Regrettably, it seems that this remains true today.

- Finally, it would be useful to know if the relative weakness of the evidence found in the systematic review supporting xylitol's value in caries reduction would lead Macquire and Rugg-Gunn ${ }^{6}$ and Edgar $^{7}$ to modify their views on the subject. While the extent of the beneficial role of sugar-free gum-containing xylitol remains under question, the principal emphasis should continue to be laid upon four key areas for oral health education: ${ }^{1}$ diet, oral hygiene, water fluoridation and dental attendance.

\section{Catherine Stillman-Lowe}

Independent oral health promotion adviser, Reading, Berkshire, UK

1. Levine RS, Stillman-Lowe CR. The Scientific Basis of Oral Health Education. 5th Edn. London: BDJ Books; 2004.

2. Kay El, Locker D. Effectiveness of Oral Health Promotion: a Review. London: Health Education Authority; 1997; 2-3.

3. Blinkhorn AS. Dental health education: what lessons have we ignored? Br Dent 1998; $184: 58-59$

4. Department of Health. Choosing Health: Making Healthy Choices Easier Executive Summary. London: Department of Health; 2004.

5. National Institute of Clinical Excellence. Dental Recall: Recall Interval between Routine Dental Examinations. London: National Institute of Clinical Excellence; 2004; clinical guideline 19.

6. Macquire A, Rugg-Gunn AJ. Xylitol and caries prevention - is it a magic bullet? Br Dent J 2003; 194:429-436.

7. Edgar WM. A role for sugar-free gum. I Clin Dent 1999; 10:89-93.

8. Moynihan PJ. Dietary advice in dental practice. Br Dent J 2002; 193:563-568

9. Watt RG, McGlone P. Prevention. Part 2. Dietary advice in the dental surgery. Br Dent | 2003; 195:27-31.

10. Rugg-Gunn AJ, Nunn JH. Nutrition, Diet and Oral Health. Oxford: Oxford University Press; 1999.

11. Daly B, Watt R, Batchelor P, Treasure E. Essential Dental Public Health. Oxford: Oxford University Press; 2002; 155-166.

12. Anderson A. Promoting Dietary Change in Low-income Communities: Assessing the Feasibility of Dietary Interventions in General Dental Practice. The Research Findings Register 2002; summary number 674 [retrieved 19 January 2005 from www.ReFeR.nhs.uk]

13. Department of Health. NHS Dentistry: Delivering Change - Report by the Chie Dental Officer (England) July 2004. London: Department of Health; 2004.

14. Health Development Agency. Helping Smokers Stop: A Guide for the Denta Team. London: Health Development Agency; 2004.

15. Eastern Health and Social Services Board. Nutrition and Dental Health Guidelines for Professionals. 3rd Edn. Belfast: Eastern Health and Social Services Board; 2000.

Evidence-Based Dentistry (2005) 6, 7-8.

doi:10.1038/sj.ebd.6400316 